\title{
Influence of surface anchoring and viscosity upon the switching behavior of twisted nematic cells
}

\author{
J. Stelzer, R. Hirning, and H.-R. Trebin \\ Institut für Theoretische und Angewandte Physik der Universität Stuttgart, Pfaffenwaldring 57, \\ D-70550 Stuttgart, Germany
}

(Received 29 March 1993; accepted for publication 14 July 1993)

\begin{abstract}
Numerical calculations are presented on the dynamics of twisted chiral nematic cells. We are emphasizing the influence of surface effects on the optical switching behavior. Apart from the anchoring strength we have taken into account viscous effects at the surface by constructing a surface dissipation function. The dynamical light transmission curve has been determined for different strengths of the surface anchoring and various viscosity parameters by computer simulation.
\end{abstract}

\section{INTRODUCTION}

The switching behavior of twisted nematic liquid crystal displays is determined not only by the visco-elastic properties of the medium itself, but also by the preparation of the display surfaces. ${ }^{1}$ By mechanical or chemical surface treatment (rubbing the glass, evaporation of a molecular layer) a preferred orientation is obtained, ${ }^{2,3}$ along which the director is minimizing the surface energy. The strength of surface anchoring (whether the director orientation is fixed or is able to turn away from the pre-orientation) is crucial for the switching characteristics.

We have evaluated the nematodynamical and Maxwell equations to calculate the light transmission of twisted and supertwisted nematic cells (TN and STN, respectively), when a voltage is suddenly applied to the cell or is taken off. Anchoring on the surfaces was taken into account by a Rapini-Papoular surface potential. Because for weak anchoring the director is able to rotate within the surface, we have included viscous surface terms, for which we established a particular surface dissipation function.

The differential equations were solved numerically. We present transmission curves for several sets of anchoring constants and surface viscosities and discuss the influence of the surface parameters on the switching behavior.

\section{THEORY}

Nematic and cholesteric liquid crystals are considered filled in between two parallel cover glasses at Cartesian coordinates $z=0$ and $z=d$. The glass separation is very small compared to the lateral extension of the glasses (micrometers versus millimeters), so we can make use of a one-dimensional geometry, i.e., all functions depend (apart from the time variable $t$ ) only on the $z$-coordinate, and there is no flow perpendicular to the surface. The director is expressed by its tilt angle $\theta$ (measured from the layer normal) and twist angle $\phi$. Thus in the one-dimensional geometry there are four unknown quantities: the angles $\theta(z, t), \phi(z, t)$ and the velocity components $v_{x}(z, t)$, $v_{y}(z, t)$.

\section{A. Material functions}

To obtain the differential equations of these functions, we describe the liquid crystal and its interaction with both external electrical fields and surfaces by phenomenological material functions. The free-energy density $\mathscr{F}$ in the bulk is taken in the form of the Frank-Oseen energy: ${ }^{4}$

$$
\begin{aligned}
\mathscr{F}= & \frac{1}{2} K_{11}(\operatorname{div} \mathbf{n})^{2}+\frac{1}{2} K_{22}\left(\mathbf{n} \cdot \operatorname{rot} \mathbf{n}-q_{0}\right)^{2} \\
& +\frac{1}{2} K_{33}(\mathbf{n} \times \operatorname{rot} \mathbf{n})^{2}+\frac{1}{2 \epsilon_{0}} \mathbf{D} \cdot \underline{\epsilon}^{-1} \cdot \mathbf{D}
\end{aligned}
$$

where $\mathbf{n}$ denotes the director, $\mathbf{D}$ the dielectric displacement, and $\underline{\epsilon}$ the dielectric tensor. $K_{11}, K_{22}, K_{33}$ are elastic constants of the liquid crystal, $q_{0}$ is the intrinsic twist due to dotation with a chiral medium.

The viscous bulk effects are described by a dissipation function $\mathscr{D}$, containing the Leslie viscosities $\alpha_{i}{ }^{5}$

$$
\begin{aligned}
\mathscr{D}= & \eta A_{i j} A_{j i}+\mu_{1} N_{i} N_{i}+\mu_{2} n_{i} n_{j} n_{k} n_{l} A_{i j} A_{k l} \\
& +\mu_{3} n_{i} n_{j} A_{j k} A_{k i}+\mu_{4} n_{i} N_{j} A_{j i} \\
A_{i j}= & \frac{1}{2}\left(\frac{\partial v_{i}}{\partial x_{j}}+\frac{\partial v_{j}}{\partial x_{i}}\right), \quad W_{i j}=\frac{1}{2}\left(\frac{\partial v_{i}}{\partial x_{j}}-\frac{\partial v_{j}}{\partial x_{i}}\right), \quad N_{i}=\dot{n}_{i}-W_{i j} n_{j}
\end{aligned}
$$

$\eta=\frac{1}{2} \alpha_{4}, \mu_{1}=\alpha_{3}-\alpha_{2}, \quad \mu_{2}=\frac{1}{2} \alpha_{1}$,

$\mu_{3}=\alpha_{5}+\alpha_{2}, \quad \mu_{4}=\alpha_{3}+\alpha_{2}$.

The dielectric anisotropy of the liquid crystal is described by the uniaxial tensor $\underline{\epsilon}^{5}$

$$
\underline{\epsilon}=\frac{1}{3} \operatorname{trace}(\underline{\epsilon}) \cdot \underline{1}+\left(\epsilon_{\|}-\epsilon_{1}\right)\left(\mathbf{n} \otimes \mathbf{n}-\frac{1}{3} \cdot \underline{1}\right)
$$

where $\epsilon_{\|}, \epsilon_{1}$ are the dielectric constants for parallel and perpendicular orientation of the director relative to the electric field.

Anchoring on the surface is expressed by the extended Rapini-Papoular potential:,

$$
\mathscr{F}^{s}=\frac{1}{2} c_{\theta} \sin ^{2}\left(\theta-\theta_{r}\right)+\frac{1}{2} c_{\phi} \sin ^{2}\left(\phi-\phi_{r}\right) .
$$


The angles $\theta_{r}, \phi_{r}$ (pretilt, pretwist) determine the preferred director orientation on the surface, the quantities $c_{\theta}, c_{\phi}$ denote the surface anchoring strength.

Viscous surface effects are described as in the bulk by a dissipation function. In constructing such a function, we assume that the viscous moments are linear in the time derivatives of the director. Hence the dissipation function should contain only terms quadratic in $\dot{n}_{i}$. We also must observe the invariance under director reversal. The preferred surface orientation $\mathbf{n}_{r}$ is taken into account by scalar products with the director and its time derivative. Thus we arrive at the following expression for the surface dissipation function:

$$
\begin{aligned}
\mathscr{D}^{s}= & \frac{1}{2} \alpha_{\|}^{s}\left(\mathbf{n}_{r} \cdot \mathbf{n}\right)^{2} \dot{\mathbf{n}}^{2}+\frac{1}{2} \alpha_{\perp}^{s}\left(\mathbf{n}_{r} \cdot \dot{\mathbf{n}}\right)^{2} \\
& +\frac{1}{2} \alpha_{\text {mix }}^{s}\left(\mathbf{n}_{r} \cdot \dot{\mathbf{n}}\right)^{2}\left(\mathbf{n}_{r} \cdot \mathbf{n}\right)^{2} .
\end{aligned}
$$

The first term is maximal when the surface director points along the preferred orientation: therefore we call its weight $\alpha_{\|}^{s}$ parallel viscosity. Since $\mathbf{n}$ has unit length, $\dot{\mathbf{n}}$ always stands perpendicular. Hence the weight of the second term $\alpha_{1}^{s}$ is denoted perpendicular viscosity, whereas the coefficient of the third summand $\alpha_{\text {mix }}^{s}$ is referred to as mixed viscosity.

\section{B. Differential equations}

The four required differential equations are the momentum balance and the Leslie-Ericksen equations, obtained by variation of the total energy, to which viscous terms are added. The mass balance for the incompressible medium ( $\operatorname{div} \mathbf{v}=0$ ) is fullfilled by the one-dimensional geometry and $v_{z}=0$.

In all dynamical equations we neglect inertial terms, as they are considerably weaker than the electric, elastic and viscous terms. ${ }^{8}$ Thus the momentum balance $(0=\rho \dot{\mathrm{v}}$ $=$ Divo) in one-dimensional geometry yields two scalar equations, namely that the components $\sigma_{z x}$ and $\sigma_{z y}$ of the stress tensor, defined ${ }^{9}$ by

$$
\sigma_{i j}=\frac{\partial \mathscr{D}}{\partial\left(\partial_{i} v_{j}\right)},
$$

are spatially constant:

$$
\begin{aligned}
\sigma_{z x}= & T_{11}(\theta, \phi) v_{x}^{\prime}+T_{12}(\theta, \phi) v_{y}^{\prime}+T_{13}(\theta, \phi) \dot{\theta} \\
& +T_{14}(\theta, \phi) \dot{\phi}=\text { const. } \\
\sigma_{z y}= & T_{12}(\theta, \phi) v_{x}^{\prime}+T_{22}(\theta, \phi) v_{y}^{\prime}+T_{23}(\theta, \phi) \dot{\theta} \\
& +T_{24}(\theta, \phi) \dot{\phi}=\text { const. }
\end{aligned}
$$

The coefficients $T_{i j}(\theta, \phi)$ are listed in the Appendix. On the surface we assume that the velocities vanish.

The Leslie-Ericksen equations for the director field are obtained by the condition of a stationary total energy $F$ which leads to separate bulk and surface equations. ${ }^{7} \mathrm{Hav}-$ ing performed the variation, we add the viscous terms (originating from the dissipation functions) to the LeslieEricksen equations and arrive at Euler-Lagrangian types of equations:

$$
\begin{aligned}
& \frac{\partial \mathscr{F}}{\partial n_{i}}-\partial_{j} \frac{\partial \mathscr{F}}{\partial\left(\partial_{j} n_{i}\right)}+\frac{\partial \mathscr{D}}{\partial \dot{n}_{i}}+\lambda n_{i}=0 \\
& \frac{\partial \mathscr{F} s}{\partial n_{i}} \mp \frac{\partial \mathscr{F}}{\partial\left(\partial_{z} n_{i}\right)}+\frac{\partial \mathscr{D}^{s}}{\partial \dot{n}_{i}}=0, \quad z=0, d \text { resp. }
\end{aligned}
$$

Their explicit form (again the coefficients are listed in the Appendix):

$$
\begin{aligned}
& A_{1}(\theta) \theta^{\prime \prime}+A_{2}(\theta) \theta^{\prime 2}+A_{3}(\theta) \phi^{\prime 2}+A_{4}(\theta) \phi^{\prime} q_{0}+E(\mathbf{D}, \theta) \\
& \quad+T_{31}(\theta, \phi) v_{x}^{\prime}+T_{32}(\theta, \phi) v_{y}^{\prime}=\gamma \dot{\theta} \\
& B_{1}(\theta) \phi^{\prime \prime}+B_{2}(\theta) \theta^{\prime} \phi^{\prime}+B_{3}(\theta) \theta^{\prime} q_{0}+T_{41}(\theta, \phi) v_{x}^{\prime} \\
& \quad+T_{42}(\theta, \phi) v_{y}^{\prime}=\gamma \dot{\phi} \\
& U_{33} \dot{\theta}_{0}+U_{34} \dot{\phi}_{0}=W_{30}, \quad z=0 \\
& U_{34} \dot{\theta}_{0}+U_{44} \dot{\phi}_{0}=W_{40}, \quad z=0 \\
& U_{55} \dot{\theta}_{d}+U_{56} \dot{\phi}_{d}=W_{50}, \quad z=d \\
& U_{56} \dot{\theta}_{d}+U_{66} \dot{\phi}_{d}=W_{60}, \quad z=d
\end{aligned}
$$

reveals a coupled set of nonlinear partial differential equations, which have to be solved numerically.

\section{Numerical procedure}

Without inertial effects the differential equations are of first order in time. We used a numerical procedure proposed by Berreman ${ }^{10}$ to solve them. The basic idea is to make the partial differential equations spatially discrete. Spatial integration of the dielectric linear material function

$$
D_{z}=\epsilon_{0} \epsilon_{z z}(\theta) E_{z}
$$

and of the momentum balance over the cell thickness yields the actual dielectric displacement and stress tensor components. These are used to obtain the actual velocity gradients from the momentum balance. The latter are inserted into the Leslie-Ericksen equations. Then we obtain a system of ordinary differential equations having only the time variable as argument: In each discretization point the time derivatives of tilt and twist angles are given, depending only on angles (and explicitly on time, if there is a time-dependent voltage). These ordinary differential equations can be integrated as an initial-value problem, by use of a standard method.

After performing the spatial discretization, all spatial derivatives have changed into difference quotients. The dielectric displacement and the stress tensor are spatially constant, but they vary in time. Hence we have to calculate them at any time step. This is performed by spatial integration from $z=0$ to $z=d$. In order to obtain the actual dielectric displacement we integrate the dielectric material function (19):

$$
D_{z}=\frac{\epsilon_{0} U}{\int d z / \epsilon_{z z}}
$$

where $U$ is the electric voltage.

In a next step the time derivatives of tilt and twist are eliminated from the momentum balance $[(9),(10)]$ by 
TABLE I. Fixed material parameters used in calculations.

$$
\begin{gathered}
\text { Volume elastic constants }[\mathrm{N}] \text { : } \\
K_{11}=1.12 \times 10^{-11} \\
K_{22}=4.91 \times 10^{-12} \\
K_{33}=9.91 \times 10^{-12}
\end{gathered}
$$

Volume viscosities $\left[\mathrm{N} \mathrm{s}^{2} / \mathrm{m}^{2}\right]$ :

$\alpha_{1}=6.5 \times 10^{-3}$

$\alpha_{2}=-79.5 \times 10^{-3}$

$\alpha_{3}=-1.2 \times 10^{-3}$

$\alpha_{4}=83.2 \times 10^{-3}$

$\alpha_{5}=46.3 \times 10^{-3}$

$\alpha_{6}=-34.4 \times 10^{-3}$

Dielectric constants (dimensionless):

$$
\begin{aligned}
& \epsilon_{\|}=8.0 \\
& \epsilon_{\perp}=3.5
\end{aligned}
$$

inserting the Leslie-Ericksen equations (13) and (14). Now we obtain the following system of equations (again the abbreviations used are to be found in the Appendix):

$$
\begin{aligned}
& \sigma_{z x}=U_{11} v_{x}^{\prime}+U_{12} v_{y}^{\prime}+W_{10} \\
& \sigma_{z y}=U_{21} v_{x}^{\prime}+U_{22} v_{y}^{\prime}+W_{20} .
\end{aligned}
$$

This system can be solved for the gradients of velocity:

$$
\begin{aligned}
& v_{x}^{\prime}=\frac{U_{22}\left(\sigma_{z x}-W_{10}\right)+U_{12}\left(W_{20}-\sigma_{z y}\right)}{U_{11} U_{22}-U_{12} U_{21}} \\
& v_{y}^{\prime}=\frac{U_{21}\left(W_{10}-\sigma_{z x}\right)+U_{11}\left(\sigma_{z y}-W_{20}\right)}{U_{11} U_{22}-U_{12} U_{21}} .
\end{aligned}
$$

Now we perform the spatial integration, noticing that the velocity components vanish on the boundaries. Thus the left sides of Eqs. (23) and (24) become equal to zero and the right sides are integrated numerically, keeping the stress components $\sigma_{z x}$ and $\sigma_{z y}$ constant. Then a linear inhomogeneous system of equations remains, which can be solved easily for $\sigma_{z x}$ and $\sigma_{z y}$.

Having determined these quantities, we simply obtain the time derivatives of the angles by substituting backward the stress components into Eqs. (23) and (24), and then placing the velocity gradients into Eqs. (13) and (14). Thus at each discretization point in the bulk we know the actual change of director orientation. On the surface Eqs.

TABLE II. Fixed cell parameters used in calculations.

$$
\begin{gathered}
\text { Cell thickness: } \\
d=5.6 \mu \mathrm{m} \text { (TN cell) } \\
d=5.9 \mu \mathrm{m} \text { (STN cell) } \\
\text { Intrinsic twist for STN cell: } \\
\text { pitch length: } p=8.85 \mu \mathrm{m} \\
\text { applied voltage: } U=4 \mathrm{~V} \\
\text { Pretilt, pretwist on the surfaces: } \\
\theta_{r}=85^{\circ} \text { (TN cell) } \\
\theta_{r}=75^{\circ} \text { (STN cell) } \\
\phi_{r}=\mp 45^{\circ} \text { (TN cell) } \\
\phi_{r}=\mp 240^{\circ} \text { (STN cell) }
\end{gathered}
$$

TABLE III. Optical parameters fixed in calculations.

$$
\begin{gathered}
\text { Refraction indices (dimensionless): } \\
\text { Ordinary: } n_{o}=1.5 \\
\text { Extraordinary: } n_{e}=1.65 \\
\text { Glass: } n_{\text {glass }}=1.53 \\
\text { Wavelength of light: } \lambda=589 \mathrm{~nm}
\end{gathered}
$$

(15) -(18) are valid. After making them spatially discrete, on each surface they form a linear system of equations that can be solved analytically for $\dot{\theta}$ and $\dot{\phi}$.

By the above algorithm we are able to calculate the change the of director everywhere in the sample, if we start with an appropriate initial configuration. The integration to the next time step can now be done by standard methods solving initial value problems. We worked with a code using the backward differentiation formulas. ${ }^{11}$

Through Eq. (5) an inhomogeneous director field gives rise to an inhomogeneous, anisotropic dielectric medium. The optical transmission through the display has been determined by solving Maxwell's equations with Berreman's $4 \times 4$-matrix method. ${ }^{12}$

\section{NUMERICAL RESULTS AND DISCUSSION}

\section{A. Parameters used in calculations}

We have calculated the time dependence of the optical transmission during switching for variable surface parameters, but fixed volume and cell parameters (Tables I, II, III).

A reasonable range of surface elastic constants has been elaborated in the article of Hirning et al. ${ }^{7}$ where numerical calculations of static transmission curves are compared with experiments. Three cases of anchoring are examined, tilt anchoring always being one order of magnitude larger than the twist anchoring (Table IV).

No theoretical or experimental estimates exist for surface viscosities. Visible effects are obtained in a range from $10^{-8} \mathrm{~N} \mathrm{~s}^{2} / \mathrm{m}$ (weak viscosity) to $10^{-6} \mathrm{~N} \mathrm{~s}^{2} / \mathrm{m}$ (strong viscosity). In all the calculations presented below a oneconstant approximation for surface viscosities is used, i.e., parallel, perpendicular and mixed viscosities are always taken with the same value.

\section{B. Dynamical behavior of TN cells}

We start with a TN cell and weak anchoring. In Figs. 1 and 2 the transmission intensity is plotted for three surface viscosities when the voltage is being switched on and off, respectively. Polarizers are crossed, thus the transmission is maximal with zero voltage. The external field de-

TABLE IV. Surface anchoring parameters used in calculations.

\begin{tabular}{ccc}
\hline \hline Kind of anchoring & $c_{\theta}[\mathrm{N} / \mathrm{m}]$ & $c_{\phi}[\mathrm{N} / \mathrm{m}]$ \\
\hline Weak & $10^{-5}$ & $10^{-6}$ \\
Medium & $10^{-4}$ & $10^{-5}$ \\
Strong & $10^{-3}$ & $10^{-4}$ \\
\hline
\end{tabular}




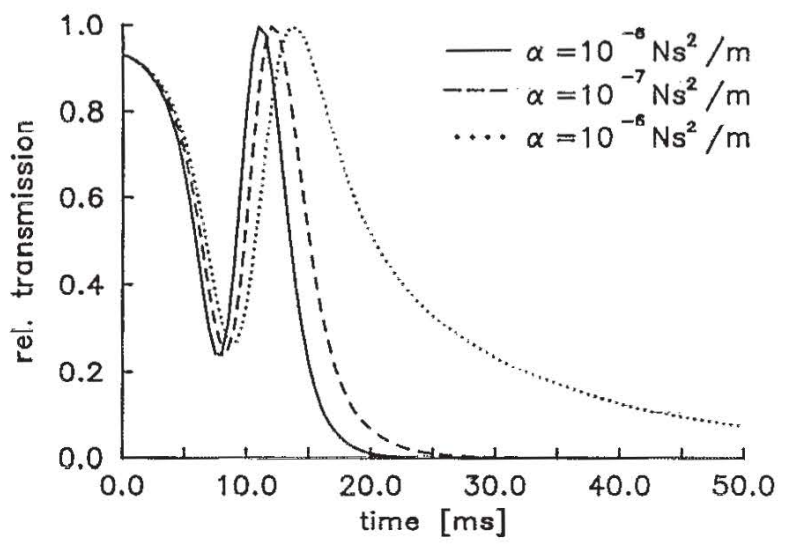

FIG. 1. TN cell switched on with weak anchoring, various viscosities.

stroys the helix and stops transmission. With weak anchoring the surface director can deviate from the preferred orientation, so that both the ordinary and the extraordinary optical mode are excited. Their interference gives rise to the pronounced transmission maxima and minima.

The director motion is due to electric, elastic, and viscous torques, appearing in the orientational balance equations (13) as terms $E(D, \theta), A(\theta)$, and $T(\theta, \phi)$. Typical values of the material parameters (Tables I, IV) reveal that the electric torque is one order of magnitude larger than the elastic torque, which exceeds the viscous torque by the same factor.

Therefore the switch-on times are ten times shorter than the switch-off times, and are influenced much less by surface viscosities than the latter.

We define the display as switched on, if the transmission intensity has dropped to $5 \%$, and as switched off, if it has gained $90 \%$. In Fig. 3 the switching times are plotted versus the surface viscosity. Remarkably, an almost linear relation is seen. The complex system of Eqs. (13) and (14) thus yields a behavior as for a simple, damped, inertia-free harmonic oscillator

$$
-\alpha \dot{\theta}+K \theta=0,
$$

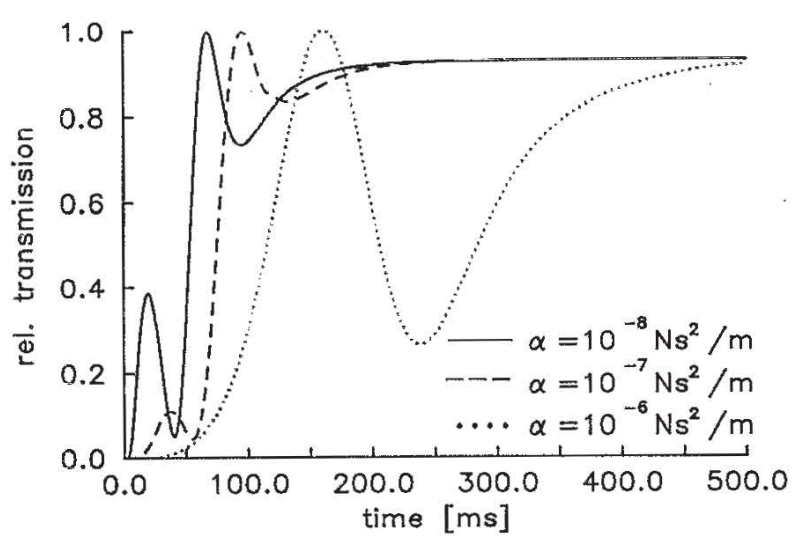

FIG. 2. TN cell switched off with weak anchoring, various viscosities.

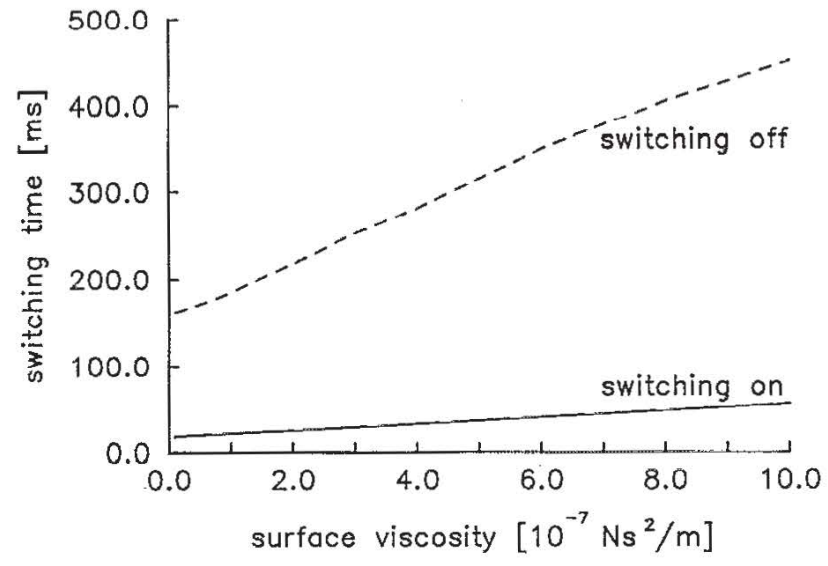

FIG. 3. TN cell, weak anchoring: switching time vs surface viscosity.

which decays exponentially: $e^{-t / \tau}$, with a time constant $\tau=\alpha / K$ proportional to the damping term $\alpha$.

In a next step the surface viscosity is kept constant $\left(\alpha=10^{-8} \mathrm{~N} \mathrm{~s}^{2} / \mathrm{m}\right)$, and the effect of various anchoring strengths on the switching behavior is studied in Figs. 4 and 5. Clearly for medium and strong anchoring the influence of surface viscosities is negligible.

\section{Dynamical behavior of STN cells}

In Figs. 6 and 7 the switching processes are shown for an STN cell with weak anchoring and various surface viscosities. The optical polarizers are parallel. To achieve destructive interference of ordinary and extraordinary light beam at zero voltage the cell thickness has been adapted according to the relation:

$$
\frac{2 \pi d}{\lambda}\left(n_{e}-n_{o}\right)=(2 v+1) \pi, \quad v=0,1,2, \ldots .
$$

After switching on the director is aligned parallel to the electric field, the liquid crystal appears as an isotropic liquid to the incident light. There is no birefringence and the optical transmission has reached its maximum value.

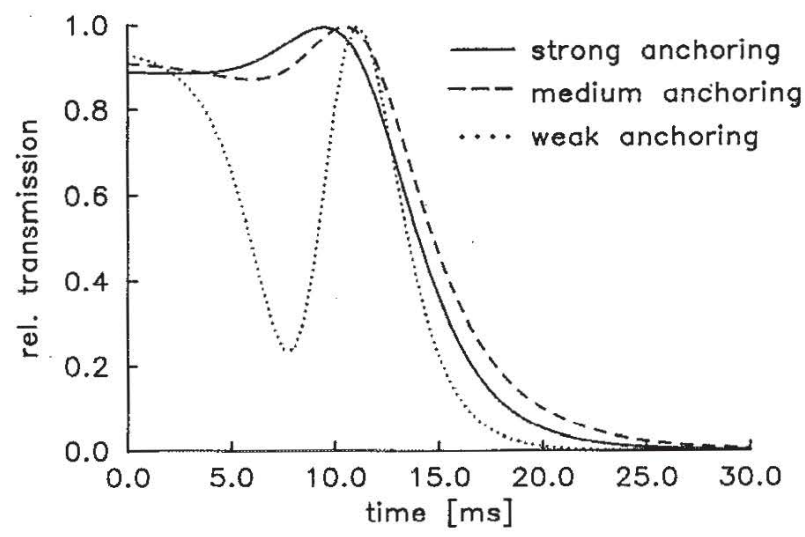

FIG. 4. TN cell switched on with weak visosity, various anchoring strengths. 


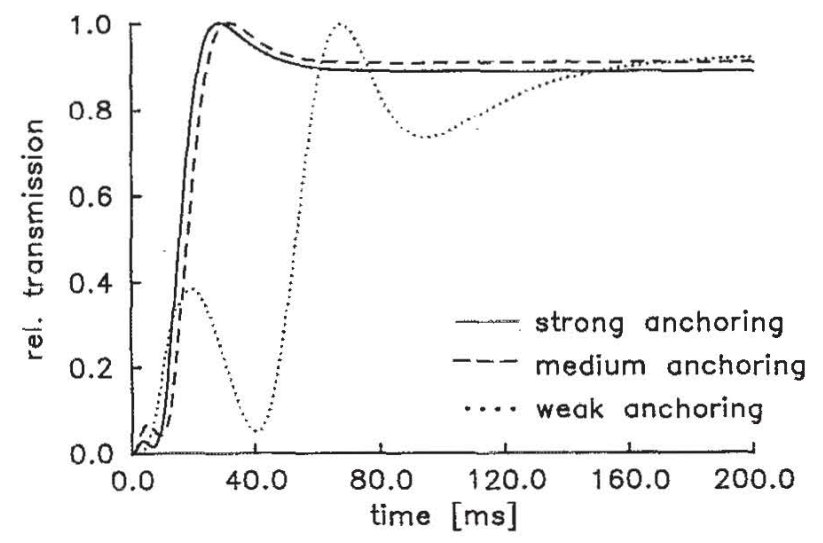

FIG. 5. TN cell switched off with weak viscosity, various anchoring strengths.

As with the TN cell, the switching time increases with the surface viscosity. Compared to that type of cell, switching off an STN cell is considerably faster, whereas the switching on is in the same time range. Because of the chiral dotation of the STN cell the director field at zero voltage is more twisted than in the TN cell. Therefore the aligned director field at full voltage is further away from the equilibrium zero voltage configuration. The elastic torques driving back the director field are stronger in the case of STN cells and reduce the time constant.

In the relation of surface viscosity and switching time (Fig. 8), we notice a deviation from linearity for small viscosities. Obviously, in this range the approximation of a harmonic oscillator is no longer valid. The effective elastic constant being larger (stronger elastic torques) than in the case of TN cells, for small viscosity a change of this quantity has a stronger effect on switching time. Thus for small values of the surface viscosity the slope of the curve is steeper.

As with TN cells we also considered the case of various anchoring strengths at constant surface viscosity $\left(\alpha=10^{-8} \mathrm{~N} \mathrm{~s}^{2} / \mathrm{m}\right)$. Similarly, only switching off is consid-

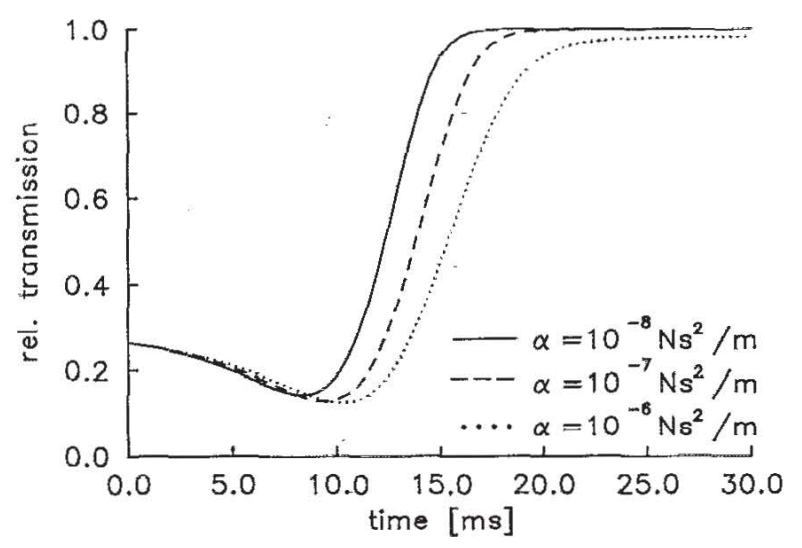

FIG. 6. STN cell switched on with weak anchoring, various viscosities.

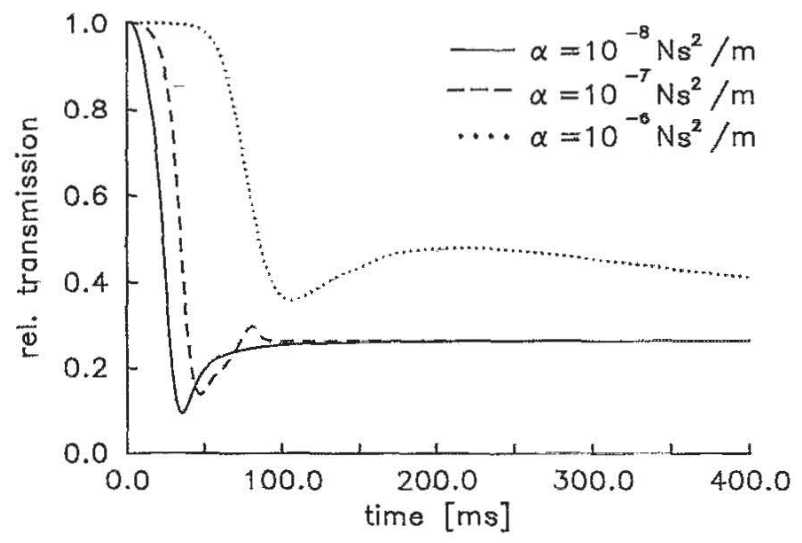

FIG. 7. STN cell switched off with weak anchoring, various viscosities.

erably affected by the anchoring, but the effect is by no means as strong as in the case of the TN cell (Figs. 9 and 10$)$.

\section{CONCLUSION}

For calculating the switching behavior of twisted and supertwisted nematic cells the liquid crystal and its interaction with an external electric field and surfaces have been described using phenomenological material functions. By means of a variation principle, partial differential equations (Leslie-Ericksen equations) have been derived. The differential equations have been solved numerically, after being converted into an initial value problem and then integrated in time. Then Maxwell's equations for an anisotropic, inhomogenous medium were solved to obtain the optical transmission. Thereby attention has been payed to the effects of various surface parameters on the switching behavior.

For TN cells the following results have been obtained:

Only with weak anchoring the surface viscosity has a noticeable influence on the switching time.

With weak anchoring a nearly linear relation between the magnitude of surface viscosity and switching time is found.

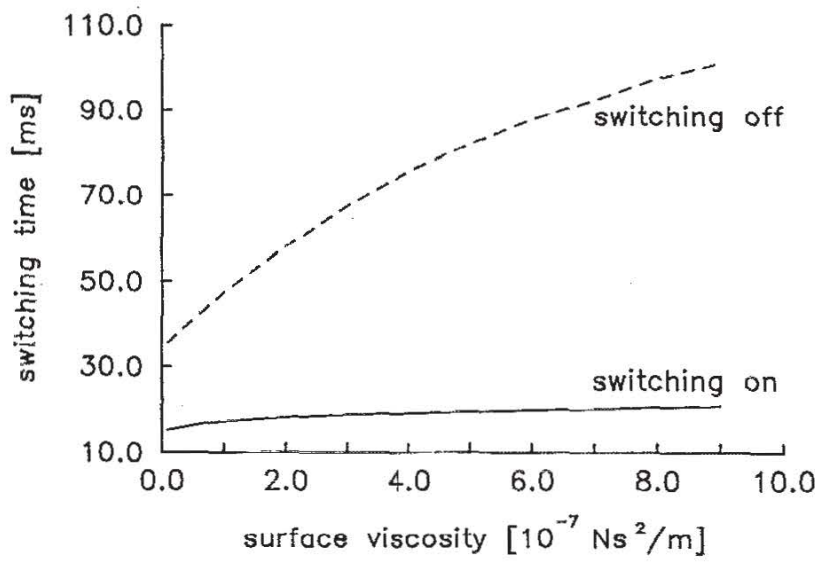

FIG. 8. STN cell, weak anchoring: switching time vs surface viscosity. 


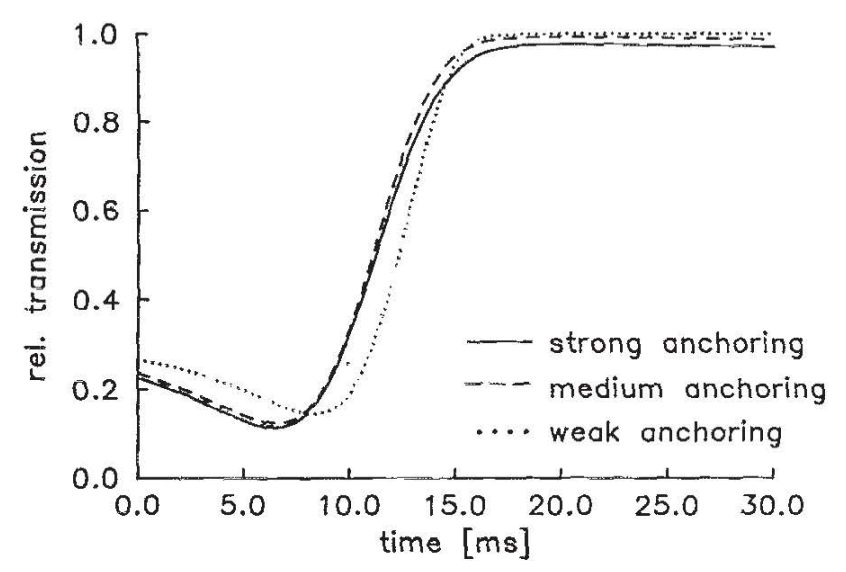

FIG. 9. STN cell switched on with weak visosity, various anchoring strengths.

The switching on is hardly, the switching off is considerably affected by the strength of surface anchoring.

The maxima and minima in the dynamics of optical transmission due to birefringence decrease with increasing strength of anchoring.

The results for STN cells can be summarized this way:

The switching off is much faster than with the TN cell, whereas the switching on is in the same range of time.

The relation between surface viscosity and switching time is a non-linear one.

As with the TN cell, only the switching off is considerably influenced by the strength of surface anchoring.

There has recently been some effort to measure surface quantities. Thus surface angles and anchoring energies have been determined experimentally. ${ }^{13-15}$

The bulk viscosities are measurable using a lowfrequency modulation method. ${ }^{16}$ An offset voltage is modulated in the frequency range of 1-100 Hertz and the time dependence of the optical transmission is measured. From this, a phase difference between voltage and transmission is obtained. By fitting the numerically calculated phase behavior to the measured one the values of some Leslie viscosities can be determined. Surface viscosities are expected

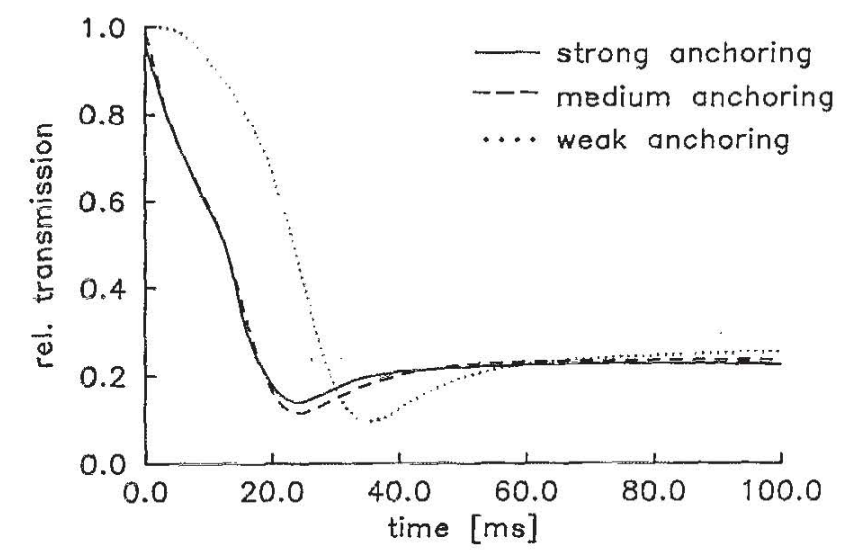

FIG. 10. STN cell switched off with weak viscosity, various anchoring strengths. to be measurable by extending this method to high offset voltages.

This work was supported by the Deutsche Forschungsgemeinschaft.

\section{APPENDIX}

All the differential equations derived in Secs. II B, II $C$, and the abbreviations introduced there are listed explicitly below.

\section{Momentum balance}

$$
\begin{aligned}
\sigma_{z x}= & T_{11}(\theta, \phi) v_{x}^{\prime}+T_{12}(\theta, \phi) v_{y}^{\prime}+T_{13}(\theta, \phi) \dot{\theta} \\
& +T_{14}(\theta, \phi) \dot{\phi}=\text { const. } \\
\sigma_{z y}= & T_{12}(\theta, \phi) v_{x}^{\prime}+T_{22}(\theta, \phi) v_{y}^{\prime}+T_{23}(\theta, \phi) \dot{\theta} \\
& +T_{24}(\theta, \phi) \dot{\phi}=\text { const. }
\end{aligned}
$$

Abbreviations used:

$$
\begin{aligned}
& T_{11}=H_{1}(\theta) \cos ^{2} \phi+H_{2}(\theta) \\
& T_{12}=H_{1}(\theta) \sin \phi \cos \phi \\
& T_{13}=H_{3}(\theta) \cos \phi \\
& T_{14}=-H_{4}(\theta) \sin \phi \\
& T_{22}=H_{1}(\theta) \sin ^{2} \phi+H_{2}(\theta) \\
& T_{23}=H_{3}(\theta) \sin \phi \\
& T_{14}=H_{4}(\theta) \cos \phi \\
& H_{1}=\left(\alpha_{1} \cos ^{2} \theta+\frac{1}{2}\left(\alpha_{3}+\alpha_{6}\right)\right) \sin ^{2} \theta \\
& H_{2}=\frac{1}{2}\left(\alpha_{4}+\left(\alpha_{5}-\alpha_{2}\right) \cos ^{2} \theta\right) \\
& H_{3}=\alpha_{2} \cos { }^{2} \theta-\alpha_{3} \sin ^{2} \theta \\
& H_{4}=\alpha_{2} \sin \theta \cos \theta \\
& H_{5}=\alpha_{2} \cot \theta .
\end{aligned}
$$

\section{Orientational balance}

$$
\begin{aligned}
\gamma \dot{\theta}= & A_{1}(\theta) \theta^{\prime \prime}+A_{2}(\theta) \theta^{2}+A_{3}(\theta) \phi^{2}+A_{4}(\theta) \phi^{\prime} q_{0} \\
& +E(D, \theta)+T_{31}(\theta, \phi) v_{x}^{\prime}+T_{32}(\theta, \phi) v_{y}^{\prime} \\
\gamma \dot{\phi}= & B_{1}(\theta) \phi^{\prime \prime}+B_{2}(\theta) \theta^{\prime} \phi^{\prime}+B_{3}(\theta) \theta^{\prime} q_{0}+T_{41}(\theta, \phi) v_{x}^{\prime} \\
& +T_{42}(\theta, \phi) v_{y}^{\prime} .
\end{aligned}
$$

Abbreviations used:

$$
\begin{aligned}
& A_{1}=K_{11} \sin ^{2} \theta+K_{33} \cos ^{2} \theta \\
& A_{2}=\left(K_{11}-K_{33}\right) \sin \theta \cos \theta \\
& A_{3}=K_{33} \sin \theta \cos \theta\left(\sin ^{2} \theta-\cos ^{2} \theta\right)-2 K_{22} \sin ^{3} \theta \cos \theta \\
& A_{4}=-2 K_{22} \sin \theta \cos \theta \\
& E=-\frac{D^{2}\left(\epsilon_{\|}-\epsilon_{\perp}\right) \sin \theta \cos \theta}{\epsilon_{0} \epsilon_{z z}(\theta)^{2}}
\end{aligned}
$$


$T_{31}=-H_{3}(\theta) \cos \phi$

$T_{32}=-H_{3}(\theta) \sin \phi$

$B_{1}=K_{22} \sin ^{2} \theta+K_{33} \cos ^{2} \theta$

$B_{2}=2 K_{33} \cot \theta\left(\cos ^{2} \theta-\sin ^{2} \theta\right)$

$B_{3}=2 K_{22} \cot \theta$

$T_{41}=H_{5}(\theta) \sin \phi$

$T_{42}=-H_{5}(\theta) \cos \phi$

$\gamma=\alpha_{3}-\alpha_{2}$

\section{Boundary equations}

$U_{33}(\theta, \phi) \dot{\theta}+U_{34}(\theta, \phi) \dot{\phi}=W_{30}\left(\theta, \theta^{\prime}\right), \quad z=0$

$U_{34}(\theta, \phi) \dot{\theta}+U_{44}(\theta, \phi) \dot{\phi}=W_{40}\left(\phi, \phi^{\prime}\right), \quad z=0$

$U_{55}(\theta, \phi) \dot{\theta}+U_{56}(\theta, \phi) \dot{\phi}=W_{50}\left(\theta, \theta^{\prime}\right), \quad z=d$

$U_{56}(\theta, \phi) \dot{\theta}+U_{66}(\theta, \phi) \dot{\phi}=W_{60}\left(\phi, \phi^{\prime}\right), \quad z=d$.

Abbreviations used:

$U_{33}=\alpha_{\|}^{s}\left(H_{6}\right)^{2}+\left(H_{7}\right)^{2} H_{12}$

$U_{34}=H_{7} H_{8} H_{12}$

$U_{44}=\alpha_{\|}^{s}\left(H_{6}\right)^{2} \sin ^{2} \theta+\left(H_{8}\right)^{2} H_{12}$

$U_{55}=\alpha_{\|}^{s}\left(H_{9}\right)^{2}+\left(H_{10}\right)^{2} H_{13}$

$U_{56}=H_{10} H_{11} H_{13}$

$U_{66}=\alpha_{\|}^{s}\left(H_{9}\right)^{2} \sin ^{2} \theta+\left(H_{11}\right)^{2} H_{13}$

$W_{30}=A_{1}(\theta) \theta^{\prime}-c_{\theta} \sin \left(\theta-\theta_{r}\right) \cos \left(\theta-\theta_{r}\right)$

$W_{40}=\left[B_{1}(\theta) \phi^{\prime}+K_{22} q_{0}\right] \sin ^{2} \theta$

$-c_{\phi} \sin \left(\phi+\frac{1}{2} \phi_{r}\right) \cos \left(\phi+\frac{1}{2} \phi_{r}\right)$

$W_{50}=-A_{1}(\theta) \theta^{\prime}-c_{\theta} \sin \left(\theta-\theta_{r}\right) \cos \left(\theta-\theta_{r}\right)$

$W_{60}=-\left[B_{1}(\theta) \phi^{\prime}+K_{22} q_{0}\right] \sin ^{2} \theta$

$-c_{\phi} \sin \left(\phi-\frac{1}{2} \phi_{r}\right) \cos \left(\phi-\frac{1}{2} \phi_{r}\right)$

$H_{6}=\sin \theta \sin \theta_{r} \cos \left(\phi+\frac{1}{2} \phi_{r}\right)+\cos \theta \cos \theta_{r}$

$H_{7}=\cos \theta \sin \theta_{r} \cos \left(\phi+\frac{1}{2} \phi_{r}\right)-\sin \theta \cos \theta_{r}$

$H_{8}=-\sin \theta \sin \theta_{r} \sin \left(\phi+\frac{1}{2} \phi_{r}\right)$

$H_{9}=\sin \theta \sin \theta_{r} \cos \left(\phi-\frac{1}{2} \phi_{r}\right)+\cos \theta \cos \theta_{r}$
$H_{10}=\cos \theta \sin \theta_{r} \cos \left(\phi-\frac{1}{2} \phi_{r}\right)-\sin \theta \cos \theta_{r}$

$H_{11}=-\sin \theta \sin \theta_{r} \sin \left(\phi-\frac{1}{2} \phi_{r}\right)$

$$
\begin{aligned}
& H_{12}=\alpha_{1}^{s}+\alpha_{\text {mix }}^{s}\left(H_{6}\right)^{2} \\
& H_{13}=\alpha_{1}^{s}+\alpha_{\text {mix }}^{s}\left(H_{9}\right)^{2} .
\end{aligned}
$$

\section{Abbreviated auxiliary quantíties}

$$
\begin{aligned}
U_{11}= & T_{11}+\frac{1}{\gamma}\left(T_{13} T_{31}+T_{14} T_{41}\right) \\
U_{12}= & T_{12}+\frac{1}{\gamma}\left(T_{13} T_{32}+T_{14} T_{42}\right) \\
U_{21}= & T_{12}+\frac{1}{\gamma}\left(T_{23} T_{31}+T_{24} T_{41}\right) \\
U_{22}= & T_{22}+\frac{1}{\gamma}\left(T_{23} T_{32}+T_{24} T_{42}\right) \\
W_{10}= & \frac{T_{13}}{\gamma}\left(A_{1} \theta^{\prime \prime}+A_{2} \theta^{\prime 2}+A_{3} \phi^{\prime 2}+A_{4} \phi^{\prime} q_{0}+E\right) \\
& +\frac{T_{14}}{\gamma}\left(B_{1} \phi^{\prime \prime}+B_{2} \theta^{\prime} \phi^{\prime}+B_{3} \theta^{\prime} q_{0}\right) \\
& T_{23} \\
W_{20} & \left.=A_{1} \theta^{\prime \prime}+A_{2} \theta^{\prime 2}+A_{3} \phi^{\prime 2}+A_{4} \phi^{\prime} q_{0}+E\right) \\
& +\frac{T_{24}}{\gamma}\left(B_{1} \phi^{\prime \prime}+B_{2} \theta^{\prime} \phi^{\prime}+B_{3} \theta^{\prime} q_{0}\right) .
\end{aligned}
$$

${ }^{1}$ B. Jérôme, Rep. Prog. Phys. 54, 391 (1991).

${ }^{2}$ B. Jérôme, A. Bosseboeuf, and P. Pieranski, Phys. Rev. A 42, 6032 (1990).

${ }^{3}$ M. Schadt, K. Schmitt, V. Kozinkov, and V. Chigrinov, Jpn. J. Appl. Phys. 31, 2155 (1992).

${ }^{4}$ F. C. Frank, Faraday Soc. Disc. 25, 19 (1958).

${ }^{5} \mathrm{G}$. Vertogen and W. H. de Jeu, Thermotropic Liquid Crystals (Springer, Berlin, 1988).

${ }^{6}$ A. Rapini and M. Papoular, J. Phys. 30C, 4 (1969).

${ }^{7}$ R. Hirning, H.-R. Trebin, M. Schmidt, and H. Schmiedel, J. Appl. Phys. 70, 4211 (1991).

${ }^{8}$ C. Z. Van Doorn, J. Appl. Phys. 46, 3738 (1975).

${ }^{9} \mathrm{~S}$. Chandrasekhar, Liquid Crystals (Cambridge University Press, Cambridge, 1980).

${ }^{10}$ D. W. Berreman, J. Appl. Phys. 46, 3746 (1975).

${ }^{11}$ E. Hairer, S. P. Nørsett, and G. Wanner, Solving Ordinary Differential Equations $I$, Springer Series in Computational Mathematics 8 (Springer, Berlin, 1987).

${ }^{12}$ D. W. Berreman, J. Opt. Soc. Am. 62, 502 (1972).

${ }^{13}$ L. M. Blinov, D. Z. Radzhabov, D. B. Subachyus, and S. V. Yablonskii, JETP Lett. 53, 238 (1991).

${ }^{14}$ H. L. Ong, J. Appl. Phys. 71, 140 (1992).

${ }^{15}$ S. Faetti and C. Lazzari, J. Appl. Phys. 71, 3204 (1992).

${ }^{16} \mathrm{H}$. Schmiedel, R. Stannarius, M. Grigutsch, R. Hirning, J. Stelzer, and H.-R. Trebin, J. Appl. Phys. 74, 6053 (1993). 